\title{
THE DOMAIN COVERED BY A TYPICALLY-REAL FUNCTION
}

\author{
A. W. GOODMAN
}

ABSTRACT. We find the largest possible domain that is covered by $f(E)$ for every typically-real function $f(z)$. In the process we obtain a set of universal typically-real functions.

1. A set of universal typically-real functions. We recall that a function

$$
f(z)=z+\sum_{n=2}^{\infty} a_{n} z^{n}
$$

regular in $E:|z|<1$, is said to be typically-real in $E$ if $f(z)$ is real for real $z$ and only for real $z$ in $E$ [5]. We let TR denote the set of all functions with the normalization (1) that are typically-real in $E$.

We first describe a particularly interesting universal ${ }^{1}$ typically-real function.

\section{THEOREM 1. The function}

$$
G(z)=\pi^{-1} \tan \left(\pi z /\left(1+z^{2}\right)\right)
$$

is in TR. In $E$, the function $G(z)$ assumes each real value exactly once, it omits $\pm i / \pi$, and it assumes every other complex value infinitely often. Further $G^{\prime}(z)$ is never zero in $E$.

Proof. To visualize the behavior of $G(z)$ we describe the surface $G(E)$. It consists of two infinite sets of half-planes, $\operatorname{Im} w>0$ and $\operatorname{Im} w<0$, each set joined by a branch point similar in nature to $\ln (w \pm i / \pi)$. One half-plane of the first set is joined to one half-plane of the second set along the real axis. For each of the other half-planes, the real axis is the boundary.

For simplicity, set $F(z)=\pi G(z)$ and let $\zeta=z /\left(1+z^{2}\right)$. Then

$$
F(z)=\tan \pi \zeta=\frac{\sin \pi \zeta}{\cos \pi \zeta}=\frac{1}{i} \frac{e^{\pi i \zeta}-e^{-\pi i \zeta}}{e^{\pi i \zeta}+e^{-\pi i \zeta}}=i \frac{1-e^{2 \pi i \zeta}}{1+e^{2 \pi i \zeta}} .
$$

Consider first the transformation $\zeta=z /\left(1+z^{2}\right)$ on the upper half of the unit disk; $E^{+}$. It is easy to see that $\zeta\left(E^{+}\right)$is the half-plane $\operatorname{Im} w>0$. The interval $[-1,1]$ goes into the interval $[-1 / 2,1 / 2]$, and the upper boundary of $E^{+}$goes into the remainder of the real axis in a one-to-one manner.

Presented to the Society, January 27, 1977; received by the editors June 1, 1976.

AMS (MOS) subject classifications (1970). Primary 10A26, 10A32, $10 \mathrm{~A} 42$.

${ }^{1} H(z)$ is a universal typically-real function (with respect to $w=a+b i$ ) if every typically-real function that omits $a+b i$ is subordinate to $H(z)$. 
Next $\eta=e^{2 \pi i \zeta}$ takes this half-plane onto the interior of infinitely many copies of the unit disk, except for the point $\eta=0$ where the infinitely many copies have a branch point of type $\ln \eta$. In this transformation the interval $-1 / 2 \leqslant \zeta<1 / 2$ goes into the boundary of the unit disk with $\eta(-1 / 2)=$ $\eta(1 / 2)=-1$. Finally $F(z)=i(1-\eta) /(1+\eta)$ carries this infinite family of disks onto an infinite family of half-planes, $\operatorname{Im} w>0$, tied together at $w=i$. The composition of these mappings carries the interval $-1<z<1$ onto the real axis on one of the infinitely many half-planes. The Schwarz-reflection principle shows that $F(z)$ carries the full disk $E$ onto a surface of the type described at the beginning of the proof. It is then obvious geometrically that $G(z) \equiv F(z) / \pi$ has all the properties described in Theorem 1. It is easy to give an analytic proof that in $E, G(z) \neq \pm i / \pi, G^{\prime}(z) \neq 0$, and $G(z)$ assumes every other value $a+b i(b \neq 0)$ infinitely often.

The function $G(z)$ can be regarded as a universal function because the surface $G(E)$ will carry $f(E)$ for any other typically-real function that omits $\pm i / \pi$. If $b \neq 0$, then an easy transformation of $F(z)$ (see the next section) gives a universal function for typically-real functions that omit $z=a \pm b i$.

2. The region covered by $f(z)$. Brannan and Kirwan [1], proved that for every $f(z)$ in TR, the domain $f(E)$ covers the disk $|z|<1 / 4$. We will determine the maximum domain $D$ covered by $f(E)$. It turns out that $D$ is not a disk, but of course it contains the disk $|z|<1 / 4$.

We begin with the simple case in which $f(z)$ omits $b i$ in $E$, where $b>0$. Throughout our discussion we use the fact that $f(E)$ is symmetric with respect to the real axis for any typically-real function. Hence we can restrict our attention to the upper half-plane $\operatorname{Im} w>0$.

Let $f(z) \in \mathrm{TR}$ and omit $b i$, where $b>0$. Then $f(z) / \pi b$ omits $i / \pi$. If $G^{-1}(w)$ denotes the inverse function of $G(z)$, defined on the surface $G(E)$, then

$$
B(z) \equiv G^{-1}(f(z) / \pi b)
$$

is regular in $E$ and satisfies the conditions of Schwarz's Lemma. Hence $f(z)=\pi b G(B(z))$,

$$
f^{\prime}(z)=\pi b G^{\prime}(B(z)) B^{\prime}(z)
$$

and at $z=0$, we have

$$
1=f^{\prime}(0)=\pi b G^{\prime}(0) B^{\prime}(0) \leqslant \pi b,
$$

since $G^{\prime}(0)=1$ and $0<B^{\prime}(0) \leqslant 1$. Further, equality can occur if and only if $B(z) \equiv z$ and $f(z) \equiv G(z)$. Thus if $f(z) \in \mathrm{TR}$ and omits $b i$ with $b>0$ then $b>1 / \pi$ and the inequality is sharp.

Now assume that $f(z) \in \mathrm{TR}$ and omits $a+b i$ where $a \neq 0$ and $b>0$. For convenience set $a+b i=\rho e^{i \alpha}$ where $0<\alpha<\pi$ and $\alpha \neq \pi / 2$ since $a \neq 0$. We let $c$ be the unique real root in $E$ of the equation

$$
G(z)=-\pi^{-1} \cot \alpha=-\pi^{-1} a b^{-1},
$$


where $G(z)$ is our universal function (2). Theorem 1 assures us that there is exactly one root in $(-1,1)$. A brief computation using $\tan \left(\pi z /\left(1+z^{2}\right)\right)=$ $\tan (\alpha-\pi / 2)$ gives

$$
z^{2}+(2 \pi /(\pi-2 \alpha)) z+1=0
$$

and hence

$$
c=\left(-\pi+2 \sqrt{\pi \alpha-\alpha^{2}}\right) /(\pi-2 \alpha) .
$$

With this $c$ set

$$
H(z)=\pi b G((z+c) /(1+c z))+a .
$$

Then the function $H(z)$ is a universal typically-real function that omits $a+b i$, and $H(0)=0$. If $H^{-1}(w)$ is the inverse function of $H(z)$ defined on the surface $H(E)$, then $H^{-1}(f(z)) \equiv B(z)$, where $B(z)$ satisfies the conditions of Schwarz's Lemma. Consequently $f(z)=H(B(z))$ and

$$
\begin{gathered}
1=f^{\prime}(0)=H^{\prime}(0) B^{\prime}(0)=\pi b G^{\prime}(c)\left(1-c^{2}\right) B^{\prime}(0) \\
=\pi b\left(1+\tan ^{2} \frac{\pi c}{1+c^{2}}\right)\left(\frac{1-c^{2}}{1+c^{2}}\right)^{2} B^{\prime}(0), \\
1=\pi b \csc ^{2} \alpha\left(\frac{1-c^{2}}{1+c^{2}}\right)^{2} B^{\prime}(0) .
\end{gathered}
$$

Since $c$ is a root of equation (8) we have

$$
\begin{aligned}
\left(\frac{1-c^{2}}{1+c^{2}}\right)^{2} & =\frac{\left(1+c^{2}\right)^{2}-4 c^{2}}{\left(1+c^{2}\right)^{2}} \\
& =\frac{[2 \pi /(\pi-2 \alpha)]^{2}-4}{[2 \pi /(\pi-2 \alpha)]^{2}}=\frac{4}{\pi^{2}}\left(\pi \alpha-\alpha^{2}\right) .
\end{aligned}
$$

We use this in (11), together with $0<B^{\prime}(0)<1$, and $b \csc \alpha=\rho$. This gives

$$
\rho \geqslant \frac{\pi}{4} \frac{\sin \alpha}{\alpha(\pi-\alpha)}, \quad 0<\alpha<\pi, \alpha \neq \pi / 2,
$$

with equality if and only if $B(z) \equiv z$. But equality is possible. When $B(z) \equiv$ $z$, then $f(z)=H(z)$.

We note that in the proof we were forced to assume that $a \neq 0, \alpha \neq \pi / 2$. But if we set $\alpha=\pi / 2$, equation (13) gives $\rho \equiv b>1 / \pi$. Further as $\alpha \rightarrow 0^{+}$ or $\alpha \rightarrow \pi^{-}$we have

$$
\lim \frac{\pi}{4} \frac{\sin \alpha}{\alpha(\pi-\alpha)}=\frac{1}{4},
$$

the Koebe constant. That $|a| \geqslant 1 / 4$ can be established by the same type of subordination argument, but it is interesting to observe that in this special case $(b=0)$ the universal functions are $F(z)=z /(1 \pm z)^{2}$, a rather abrupt change from $G(z)$ given by (2). We have proved 
TheOREM 2. Let $D$ be the largest domain such that $D \subset f(E)$ for every $f(z)$ in TR. Then the upper boundary of $D$ is given in polar coordinates $(\rho, \theta)$ by

$$
\rho=(\pi \sin \theta) / 4 \theta(\pi-\theta),
$$

and the lower boundary is obtained by reflecting this curve in the real axis. The points $w= \pm 1 / 4$ are also part of the boundary of $D$.

3. Comments. M. S. Robertson [4] has proved that if $f(z) \in \mathrm{TR}$, then it has an integral representation

$$
f(z)=\frac{1}{\pi} \int_{0}^{\pi} \frac{z}{1-2 z \cos t+z^{2}} d \mu(t)
$$

where $\mu(t)$ is nondecreasing on $[0, \pi]$ and

$$
\int_{0}^{\pi} d \mu(t)=\pi
$$

Since $G(z)$ has such unusual properties it would be interesting to have $\mu(t)$ explicitly for this function. For one thing, we could compute the coefficients in the power series for $G(z)$-coefficients that seem to be difficult to compute directly from (2).

It is known [5] that if $f(z)$ is a normalized typically-real function, then

$$
g(z) \equiv\left[\left(1-z^{2}\right) / z\right] f(z)=1+p_{1} z+p_{2} z^{2}+\ldots
$$

is in $P$, the set of normalized functions with $\operatorname{Re} g(z)>0$ in $E$. One might ask for the largest domain covered by every function in this set. Here the corresponding question is trivial because for the set $P$, the set $D$ that is always covered consists of the single point $w=1$ (and does not form a domain). Even if we consider the subset of univalent functions in $P$, we get the same trivial answer: $D=\{1\}$.

The largest domain $D$ covered by $f(E)$ for every $f(z)$ in a certain set $M$ of normalized functions has been treated by other writers. Classical distortion theorems give the disk $|w|<1 / 4$ if $M$ is the set $S$ of normalized univalent functions, and $|w|<1 / 2$ for the subset of convex functions. Goodman and Saff [2] found $D$ for the subset of $S$ of functions convex in the direction of the imaginary axis. Further McGregor [3] has determined $D$ for subsets of $S$ for which all the coefficients are real and: (a) $f(z)$ is starlike, (b) $f(z)$ is convex, and (c) $f(z)$ is convex in the direction of the imaginary axis.

The largest domain covered by $f(E)$ for every $f(z)$ in $M$ is sometimes referred to as the Koebe domain for the set $M$. For more references to papers on Koebe domains the reader should consult Bernardi's Bibliography of schlicht functions, Part I (1966) and Part II (1977), Courant Institute of Mathematical Sciences, New York University, New York.

\section{REFERENCES}

1. D. A. Brannan and W. E. Kirwan, A covering theorem for typically real functions, Glasgow Math. J. 10 (1969), 153-155. MR 40\#7431.

2. A. W. Goodman and E. B. Saff, On univalent functions convex in one direction (to appear). 
3. M. T. McGregor, On three classes of univalent functions with real coefficients, J. London Math. Soc. 39 (1964), 43-50.

4. M. S. Robertson, On the coefficients of a typically-real function, Bull. Amer. Math. Soc. 41 (1935), 565-572.

5. W. Rogosinski, Über positive harmonische Entwicklungen und typisch-reele Potenzreihen, Math Z. 35 (1932), 93-121.

Département de Mathímatiques, Universtrí de Parus ViI, Paris, France

Current address: Department of Mathematics, University of South Florida, Tampa, Florida 33620 